\title{
Lean and obese microbiota: differences in in vitro fermentation of food-by-products
}

\author{
C. Bussolo de Souza ${ }^{1}$, S.M.I. Saad ${ }^{2}$ and K. Venema ${ }^{1,3^{\prime \prime}}$ \\ ${ }^{1}$ Maastricht University - campus Venlo, Centre for Healthy Eating \& Food Innovation, Villafloraweg 1, 5928 SZ Venlo, the \\ Netherlands; ${ }^{2}$ University of São Paulo, School of Pharmaceutical Sciences, Dept. Biochemical and Pharmaceutical Technology, \\ Av. Professor Lineu Prestes 580, 05508-000 São Paulo, Brazil; ${ }^{3}$ Beneficial Microbes Consultancy, Johan Karschstraat 3 , \\ 6709 TN Wageningen, the Netherlands; k.venema@maastrichtuniversity.nl
}

Received: 27 July 2020 / Accepted: 1 November 2020

(c) 2021 Wageningen Academic Publishers

OPEN ACCESS (1) (1)(2) RESEARCH ARTICLE

\begin{abstract}
The aim of the study was to investigate the potential prebiotic effects of food-by-products (cassava bagasse $(\mathrm{n}=3)$, orange bagasse $(\mathrm{n}=2)$ and passion fruit peel $(\mathrm{n}=3))$ using an in vitro model simulating the proximal colon, and to assess possible differences in fermentation when using faecal microbiota from lean or obese people. Fermentation of the by-products was compared to a control medium and the prebiotic inulin. The effects of the by-products on the dynamics of the gut microbiota differed according to the type of microbiota, as well as the type of by-product used. Principal Coordinate Analysis of the microbiota showed evidence of a clear separate clustering of lean and obese microbiota before the addition of substrates, which disappeared after fermentation, and instead, distinct clusters due to primary carbohydrate composition of the by-products (starch, fructan and pectin) were present. This is evidence that the substrates drove the obese microbiota to a healthier profile, more similar to that of the lean microbiota. Cassava bagasses enriched the beneficial genus Bifidobacterium in the obese microbiota. The production of total SCFA by cassava bagasses by the obese microbiota was higher than for control medium and inulin. Orange bagasses stimulated the growth of the butyrate-producing genus Coprococcus. Passion fruit peels were poorly fermented and generated negligible amounts of intermediate metabolites, indicating slow fermentation. Nevertheless, passion fruit peel fermentation resulted in a microbiota with the highest diversity and evenness, a positive trait regarding host health. In conclusion, the use of food-by-products could be an important step to tackle obesity and decrease the waste of valuable food material and consequently environmental pollution. They are an inexpensive and non-invasive way to be used as a dietary intervention to improve health, as they were shown here to drive the composition of the obese microbiota to a healthier profile.
\end{abstract}

Keywords: cassava bagasse, orange bagasse, passion fruit peel, gut microbiota, prebiotic

\section{Introduction}

The problem of overweight and obesity has reached an incredible dimension worldwide, and some of the consequences are the increased risk for several diseases, such as cardiovascular disease, different types of cancer and more recently, as a factor for disease severity of the novel coronavirus (COVID-19) (Simonnet et al., 2020; WHO, 2020).

The gut microbiota is emerging as a possible tool to be used in this battle against obesity. In this context, host diet has a direct influence on microbiota composition (Shin et al.,
2019), and is considered an important way to modulate the gut microbiota (Scott et al., 2013). Indigestible products that reach the colon are the main substrates for the gut microbiota, and among them, dietary fibres constitute an important source of energy for these microorganisms (Martens et al., 2014; Martinez et al., 2015). Fibres that can selectively be used by the host gut microbiota and confer health benefits to the host are the so-called prebiotics (Gibson et al., 2017).

The end products of fibre fermentation by the gut microbiota include the beneficial metabolites short chain fatty acids (SCFA), mainly acetate, propionate and butyrate, 
as well as lactate (Kovatcheva-Datchary et al., 2009). One of the proposed mechanisms by which SCFA may protect against obesity is through the activation of free fatty acid receptor (FFAR) 2 and 3 (Nohr et al., 2013) by SCFA. FFAR 2 and 3 are responsible for triggering the release of the satiety hormones glucagon-like peptide-1 (GLP1) and peptide YY (PYY) by L-cells (Nohr et al., 2013; Samuel et al., 2008; Tolhurst et al., 2012), and in that way contribute to energy balance. Nowadays, however, the food industry is producing more and more ultra-processed foods by which several components from the raw material are removed and discarded, generating by-products, such as fruit and vegetable peels. These by-products are mostly underused and their inappropriate disposal in the environment contributes to pollution (Pandey et al., 2000). The food-by-products are chemically treated and then disposed in the environment without any type of cleaning, and consequently they are a source of pollution (in the case of cassava bagasse there is leaching of cyanides), and considered an environmental hazard, unlike composting. At the same time, the consumption of ultra-processed foods that lack important nutrients, like fibres, but instead are filled with high density energy ingredients (simple sugars/ fats), is contributing to the increased incidence of diseases related with bad eating habits, especially obesity (Hall et al., 2019; Louzada et al., 2015).

A proper utilisation of these by-products, such as to incorporate them back into food products, for example as functional foods, could represent one step to decrease their wastage and increase fibre intake. This interconnected scenario of lack of fibre consumption, modulation of the gut microbiota through ingestion of fibres and the use of fibre-rich by-products from the food industry seems durable with regards to a favourable human health and environmentally friendly reuse of wasted food. However, before incorporating these by-products into food, it is necessary to characterise them and to study their possible health effects. We have previously characterised the byproducts from the Brazilian food industry used here cassava bagasse, orange bagasse and passion fruit peel - regarding their chemical composition and digestibility (Bussolo de Souza et al., 2018). Interestingly, the results showed differences in composition of the same by-product from different suppliers, which can have an influence on the health effects when consuming these fibres.

The aim of the present study was to investigate the potential prebiotic effects of selected food-by-products using an in vitro model that closely simulates the proximal colon (TNO in vitro model of the colon [TIM-2]) and assess possible differences in fermentation when using a gut microbiota from lean or obese people.

\section{Material and methods}

\section{Substrates}

In total, 8 substrates (plus 2 controls) were tested for the fermentation experiments. Three different cassava bagasses (CB) were tested, CB1and CB2 were from the same supplier but different batches (Grazimara, Biguaçu, Brazil), CB3 was from Lorenz (Indaial, Brazil), all in the form of powder. Two samples of orange bagasses $(\mathrm{OB})$ were tested, both in the form of powder - OB1 from Chá\&Cia (Jacareí, Brazil), OB2 from a store with bulk sale (Florianópolis, Brazil). Three passion fruit peels (PFP) were tested, all in the form of powder - PFP1 from Chá\&Cia, PFP2 from Phytomare (Governador Celso Ramos, Brazil) and PFP3 from the same store as OB2 (Florianópolis). According to the manufacturers, only the peels were used, seeds were disposed. Inulin from chicory root was provided by its manufacturer (Orafti, Amsterdam, the Netherlands) and it was used as positive control (purity $\geq 90 \%$, degree of polymerisation $>10$ ). Simulated ileal effluent medium (SIEM) was used as control and it mimics the indigestible fibres from western diet (Sáyago-Ayerdi et al., 2020). More detailed information about the substrates was described elsewhere (Bussolo de Souza et al., 2018) and summarised in Supplementary Table S1.

\section{Dynamic in vitro model of the colon (TIM-2)}

TIM-2 is a validated, dynamic in vitro model that closely mimics the proximal colon, extensively described by Minekus et al. (1999) (Figure 1). Briefly, this computercontrolled system simulates several features from the human large intestine, such as temperature (kept at $37^{\circ} \mathrm{C}$ ), $\mathrm{pH}$ (kept at 5.8) and anaerobic environment (by constant flushing with gaseous nitrogen). Water and fermentation products are removed through a dialysis system, and the speed of the dialysis fluid was set at $1.5 \mathrm{ml} / \mathrm{min}$. This prevents accumulation of microbial metabolites which would otherwise lead to inhibition or even death of the microbiota. Mixing and transport of intestinal contents is achieved through the simulation of peristaltic movements.

\section{Origin of the gut microbiota}

The gut microbiota used for the TIM-2 experiments consisted of active pooled faecal material from (1) 8 healthy lean volunteers (body mass index $(B M I)=20 \pm 1.48 \mathrm{~kg} / \mathrm{m}^{2}$, male: $\mathrm{n}=4$, female: $\mathrm{n}=4$, age range $25-42$ years) and referred to as 'lean microbiota', or (2) 7 obese but otherwise healthy volunteers $\left(B M I=32 \pm 1.17 \mathrm{~kg} / \mathrm{m}^{2}\right.$, male: $\mathrm{n}=3$, female: $\mathrm{n}=4$, age range $29-68$ years) denoted as 'obese microbiota'. Lean volunteers were recruited from TNO (Zeist, the Netherlands) and obese volunteers from Maastricht University Medical Centre (Maastricht, the Netherlands). Volunteers received oral and written explanation about 


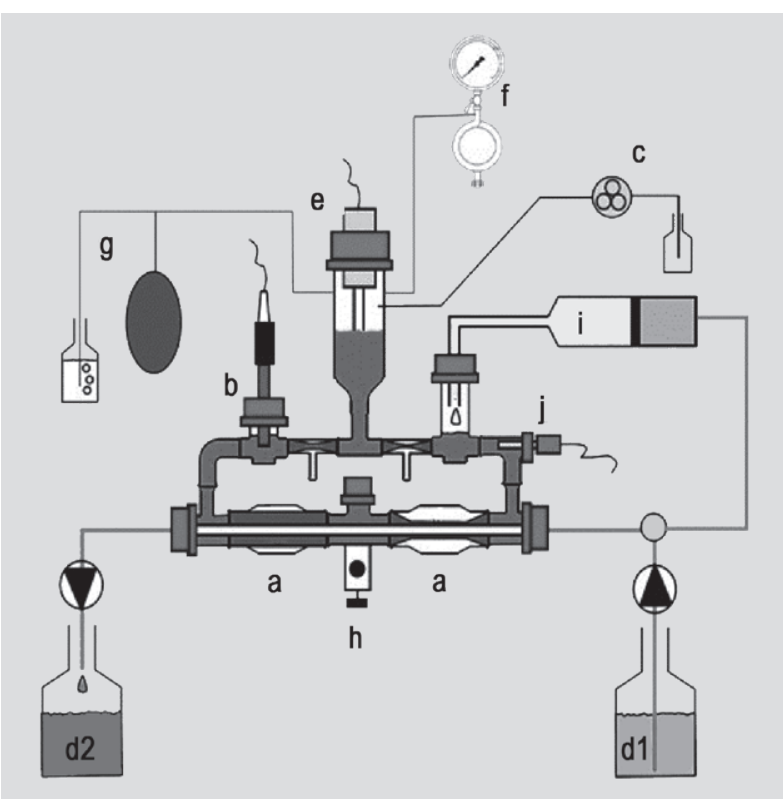

Figure 1. Schematic representation of the TIM-2 system: (a) peristaltic compartments with a dialysis membrane inside, (b) $\mathrm{pH}$ sensor, (c) $\mathrm{NaOH}$ secretion, (d) dialysate system (d1=dialysate in, d2=dialysate out), (e) level sensor, (f), gaseous $\mathrm{N}_{2}$ inlet, (g) gas outlet, (h) sampling port, (i) feeding syringe with test compound, and (j) temperature sensor. Adapted from Rehman et al. (2012).

the study and how to proceed with faeces collection. Prior to their participation, each person provided an informed consent. The exclusion criteria for both groups included the use of antibiotics, probiotics, prebiotics and laxatives in the 3 months preceding donation. Faecal samples were selfcollected: volunteers were instructed to defecate in a bag and immediately place it into a gastight plastic jar containing anaerobic packs (AnaeroGen ${ }^{\mathrm{m}}$, Oxoid, Cambridge, UK). Faeces were mixed in an anaerobic cabinet $\left(80 \% \mathrm{~N}_{2}, 10 \%\right.$ $\mathrm{CO}_{2}, 10 \% \mathrm{H}_{2}$ ) as described by Aguirre et al. (2015). The faecal material was subsequently aliquoted, snap-frozen in liquid nitrogen and stored at $-80^{\circ} \mathrm{C}$. This inoculum was thawed by $1 \mathrm{~h}$ in a $37^{\circ} \mathrm{C}$ water bath before its introduction to the TIM-2 system (Aguirre et al., 2016).

\section{Experimental protocol and study design}

Before the addition of the standardised microbiota to the system, TIM-2 was flushed with gaseous nitrogen for $3 \mathrm{~h}$ to create a complete anaerobic environment. Afterwards, the system was inoculated with approximately $30 \mathrm{ml}$ of the standardised microbiota and $90 \mathrm{ml}$ of dialysate (described by Aguirre et al., 2015), yielding a total of $120 \mathrm{ml}$ (total volume of the system). The microbiota was fed with SIEM $(2.5 \mathrm{ml} / \mathrm{h})$ and adapted to the system for $16 \mathrm{~h}$. After the adaptation period, the feeding system was stopped and a $2 \mathrm{~h}$ starvation period allowed the microbiota to ferment all remaining carbohydrates present in the model. Subsequently, samples were collected at time zero $(\mathrm{t} 0 \mathrm{~h})$ after which the substrates were added. From this point onwards, a 3-day experimental period started, and the microbiota received the standard feeding (SIEM, referred to from now on as control), or this medium but without the carbohydrate sources, which was substituted by the substrates - inulin, cassava bagasse, orange bagasse or passion fruit peel. SIEM contained 7.5 $\mathrm{g} /$ day of carbohydrates and the same amount of substrates was added. Samples of simulated lumen and spent dialysate (dialysate out) were taken at the following times: $0(\mathrm{t} 0 \mathrm{~h})$, 24. $(\mathrm{t} 24 \mathrm{~h}), 48(\mathrm{t} 48 \mathrm{~h})$ and $72 \mathrm{~h}(\mathrm{t} 72 \mathrm{~h})$ and stored $\left(-80^{\circ} \mathrm{C}\right)$ until analysis. Four TIM-2 units were run in parallel. The experimental set-up can be viewed in Figure 2.

\section{SCFA, BCFA and organic acids quantification in simulated lumen and dialysate out samples}

Simulated lumen and dialysate out samples from TIM-2 were analysed by Brightlabs (Venlo, the Netherlands) to quantify SCFA (acetate, propionate and butyrate), branched chain fatty acids (BCFA) (iso-butyrate and iso-valerate), as well as organic acids (valerate, caproate, lactate, succinate and formate), described in detail elsewhere (Larsen et al., 2019b).

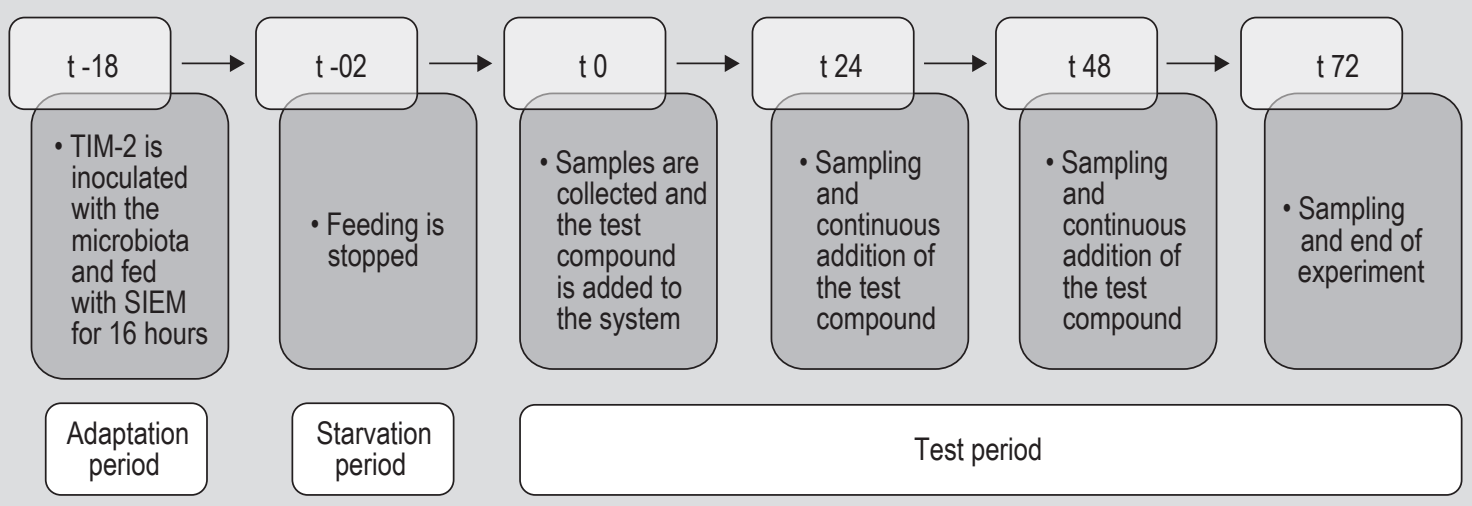

Figure 2: Study design with timeline for TIM-2 experiments. 


\section{Energy extraction}

Energy extraction in the form of SCFA was calculated using the following values for acetate, propionate, butyrate and lactate, respectively: 209, 367, 524 and $326 \mathrm{kcal} / \mathrm{mol}$ (Van der Kamp et al., 2010).

\section{Microbiota composition characterisation}

Genomic DNA isolation from TIM-2 samples (simulated lumen, $\mathrm{t} 0 \mathrm{~h}$ and $\mathrm{t} 72 \mathrm{~h}$ ) was performed using standard molecular biology kits from ZYMO (ZYMO Research, Co., Irvine, CA, USA) by Baseclear (Leiden, the Netherlands). PCR amplification of the 16S rRNA gene (V3-V4 region), barcoding and library preparation were also performed by Baseclear. Short paired-end sequence reads were generated using the Illumina MiSeq system (San Diego, CA, USA) and converted into FASTQ files using the BCL2FASTQ pipeline version 1.8.3. Quality trimming was applied based on Phred quality scores. Microbial analyses were performed with Quantitative Insight Into Microbial Ecology (QIIME) software package (version 1.9.0) (Caporaso et al., 2010). The sequences were classified using Greengenes (version 13.8) as a reference $16 \mathrm{~S}$ rRNA gene database.

\section{Data analyses}

Experiments were performed in duplicate $(n=2)$ per substrate and per microbiota (lean or obese), except for control ( $n=3$ with lean microbiota and $n=4$ with obese microbiota) and inulin $(n=3)$. Results are displayed as average. $\mathrm{R}$ (version 3.1.3) was used in RStudio for statistical analyses for gut microbiota composition. For SCFA analyses, comparison between the two groups (lean and obese) and between substrates and control was performed using t-test with significance $P<0.05$. For microbiota composition analyses, differences in multiple categories were tested with Kruskal-Wallis, with $P$-values $P<0.05$ set to be significantly different. Dunn post hoc test was used to find differences between groups. Spearman correlation was calculated between the relative abundance of operational taxonomic units (OTUs) and continuous variables. Multiple comparisons were corrected using the false discovery rate (FDR), and q-values (adjusted $P$-values) were considered significantly different at a stringent cut-off of $<0.05$. Permutational multivariate analysis of variance (PERMANOVA; (Anderson, 2017)) was performed to test the significance of beta diversity (weighted and unweighted UniFrac) between lean and obese microbiota for $\mathrm{t} 0 \mathrm{~h}$, and between primary substrates for $\mathrm{t} 72 \mathrm{~h}$ in QIIME. Changes in microbial composition were assessed through the difference of their initial relative abundance and final abundance ( $\mathrm{t} 72$ $\mathrm{h}$ - t0 h), and compared to control. Linear discriminant analysis effect size (LEfSe) (Segata et al., 2011) was used to find biomarkers between groups using relative abundances from the OTU tables generated in QIIME.

\section{Results and discussion}

\section{Obese microbiota do not always produce more total SCFA and consequently do not extract more energy than the lean microbiota}

The amount of SCFA produced by the lean and obese microbiota was dependent on the substrate tested, and demonstrated that the obese microbiota not always generated more SCFA than the lean microbiota (Figure 3 and Supplementary Figure S1). The same was the case for energy extraction in the form of SCFA that was also dependent of the test compound, and not always related to the type of microbiota (Supplementary Figure S2). This was unexpected, since a pioneering study showed that the gut microbiota from obese individuals extracted more energy than lean individuals (Turnbaugh et al., 2006). For 5 out of 10 substrates the obese microbiota was able to produce more total SCFA than the lean, although when comparing the total amount of SCFA produced by the lean and obese microbiota (from all substrates), the differences were not significant $(P>0.05)$. Similar results are available in the literature. In vitro fermentation of diverse types of fibres showed that production of SCFA and consequently energy extraction was dependent of the test compound used, not the type of inoculum (lean or obese) (Aguirre et al., 2014), which is in accordance with our results.

The role of SCFA in obesity, either protective or causative, remains to be elucidated. However, it is worth noting that when detrimental aspects are related to SCFA, this is generally present when there is a dysbiosis - related to a certain disease or inappropriate life style. In turn, these aspects affect normal microbiota functioning compositionally and metabolically speaking (Turnbaugh et al., 2006). Under 'normal' conditions (healthy state), it has to be acknowledged that SCFA are essential in maintaining the equilibrium in host health through regulation and participation in several metabolic pathways. The effects of individual SCFA are described in the sections of each test compound.

\section{Primary composition of substrates drive important changes in microbiota composition}

Principal coordinate analysis (PCoA) showed a clear clustering of lean and obese microbiota before the addition of substrates ( $\mathrm{t} 0 \mathrm{~h}$ ), in both weighted and unweighted UniFrac analyses (Figure 4A and 4B). PERMANOVA analysis demonstrated that this difference was significant between lean and obese (weighted: $P=0.002$; unweighted: $P=0.001$ ). At $\mathrm{t} 72 \mathrm{~h}$, this clustering disappeared, and instead, distinct clusters by primary composition of the substrates (starch as present in cassava bagasses and control, fructan in inulin or pectin present in orange bagasses and passion fruit peels), and no longer a distinction between lean and obese 


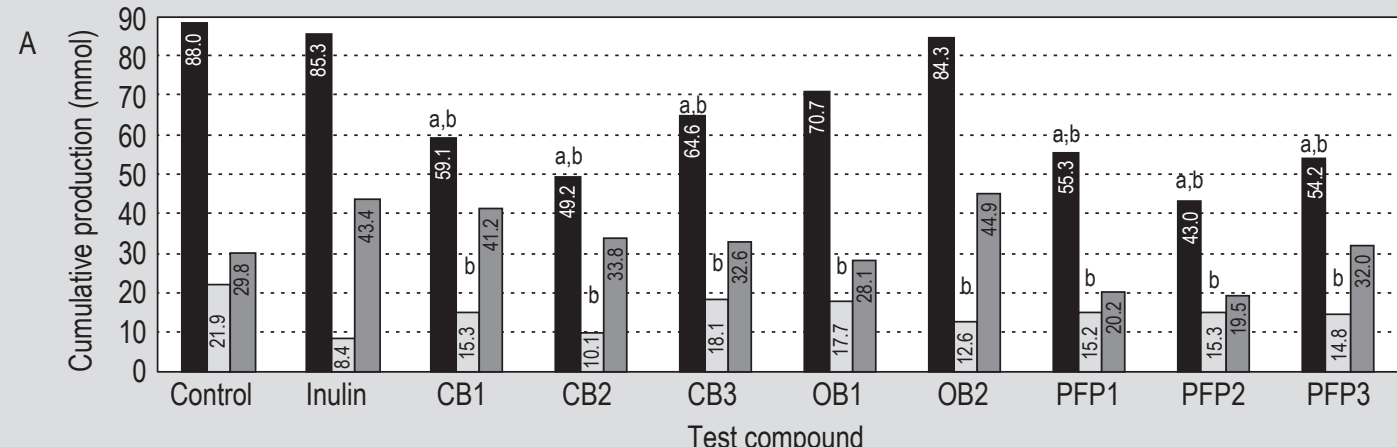

Acetate

$\square$ Propionate

B

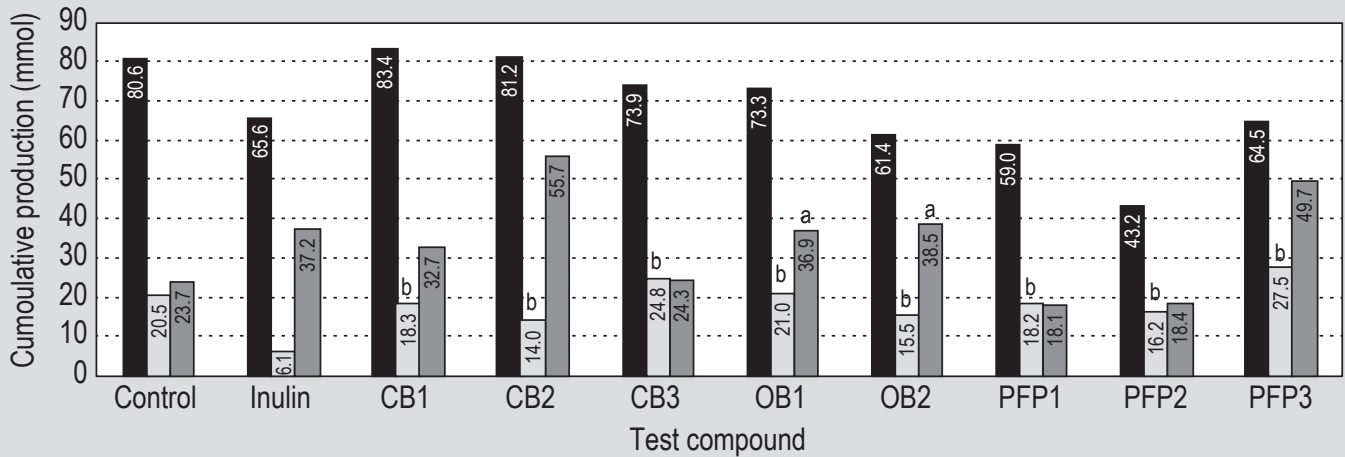

$\square$ Butyrate

Figure 3. Cumulative production of short chain fatty acids (acetate, propionate and butyrate) by the lean (A) and obese (B) microbiota at $\mathrm{t} 72 \mathrm{~h}$ with different substrates. $\mathrm{CB}=$ cassava bagasse; $\mathrm{OB}=$ orange bagasse; $\mathrm{PFP}=$ passion fruit peel.
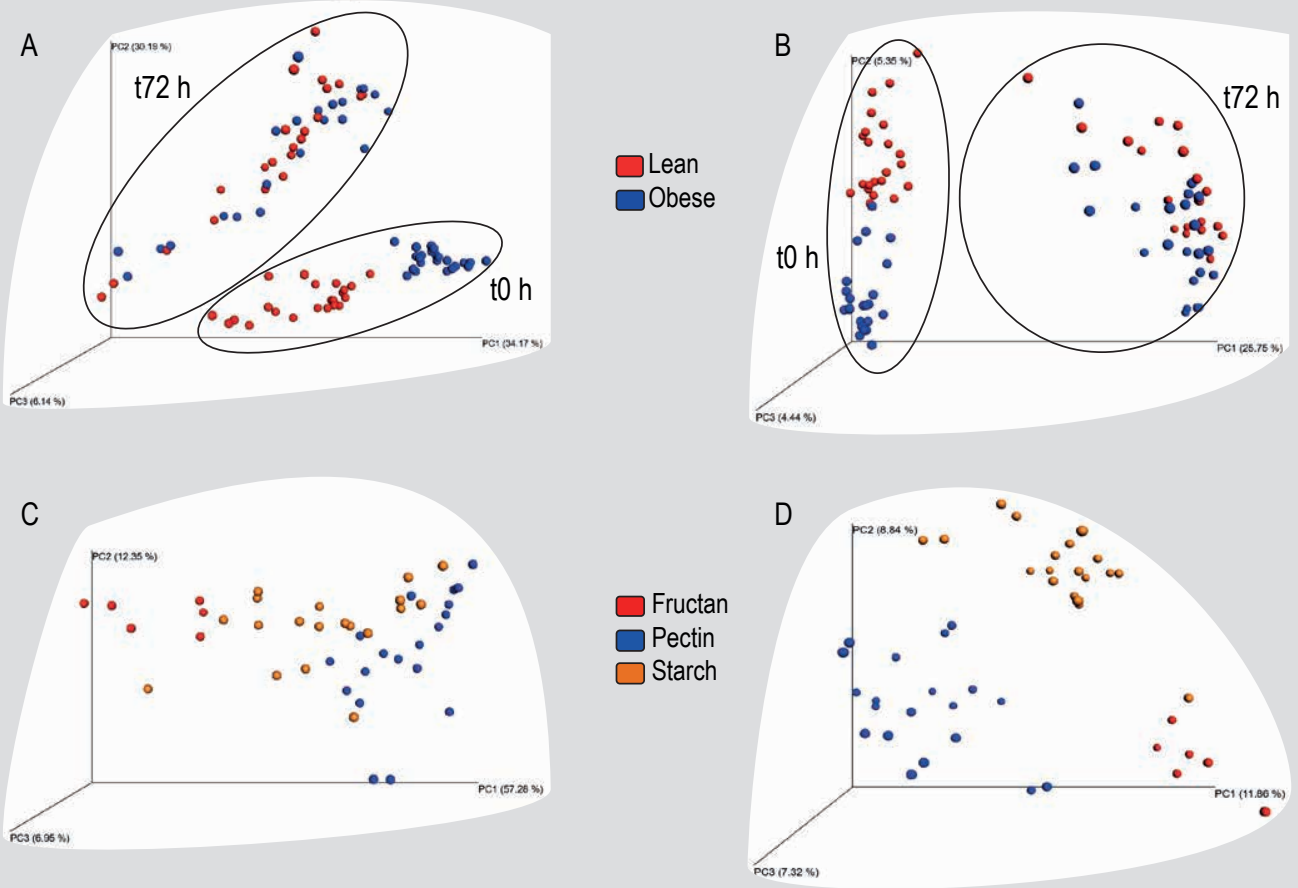

Figure 4. Principal coordinate analysis plots of the microbiota after $72 \mathrm{~h}$ fermentation period in TIM-2. Samples are plotted on the first three Principal Coordinates (PC1, PC2 and PC3). (A) Weighted and (B) unweighted UniFrac showing 2 different clusters among lean and obese samples at to $\mathrm{h}$, which disappeared after $72 \mathrm{~h}$ fermentation period. (C) Weighted and (D) unweighted UniFrac showing that at $\mathrm{t} 72 \mathrm{~h}$, samples were clustered according to their primary substrate (fructan, pectin or starch). 
microbiota, was present (Figure 4C and 4D for weighted and unweighted UniFrac). At $772 \mathrm{~h}$, the unweighted UniFrac between lean and obese was still significantly different $(P=0.001)$, but not significantly different in the weighted $(P=0.235)$.

For t72 h, the overall difference for 'primary substrate' was significant (weighted: $P=0.001$; unweighted: $P=0.001$ ). For the individual substrates: fructan vs the others (weighted: $P=0.001$; unweighted: $P=0.001$ ), pectin vs the others (weighted $P=0.001$; unweighted: $P=0.001$ ); starch vs the others (weighted: trend for significance $P=0.053$; unweighted: $P=0.001$ ). This is evidence that the substrates drove the obese microbiota to a healthier profile, that is more similar to that of the lean microbiota, with the stimulation of commensals in the simulated lumen, among them, health promoting bacteria (see below). The Shannon index showed that fermentation of PFP3, OB1 and PFP1 stimulated the growth of more bacterial species (abundance and evenness) (Supplementary Figure S3). The effects that fermentation of each test compound had on the microbial composition will be discussed below for some selected genera for which effects were observed.

\section{Cassava bagasses stimulated the beneficial genus Bifidobacterium in the obese microbiota}

In our study, for the lean microbiota, all tested compounds (except inulin) caused a decrease in Bifidobacterium when compared to the control (Figure 5A). LEfSe analysis showed that Bifidobacterium is a discriminate feature for fructan (present in inulin), in the lean microbiota (Figure 6). In the obese microbiota, nonetheless, CB1 and CB2 were the only substrates (apart from inulin) that were capable of stimulating the growth of this genus (Figure 5B). Even though Bifidobacterium ferments mostly mono- and

\section{A \#OTU ID}

$f$ _Bifidobacteriaceae;g__Bifidobacterium $f$ Coriobacteriaceae; $g$ f_Coriobacteriaceae; g_Collinsella f_Bacteroidaceae; $g$ _Bacteroides $f$ _Prevotellaceae; $g$ __Prevotella $f$ Enterococcaceae;g_Enterococcus f_Lactobacillaceae; g_Lactobacillus f_Streptococcaceae;g_Streptococcus o_Clostridiales; $f_{-}$*

$f$ _Clostridiaceae, $g$

$f$ Lachnospiraceae; Othe f_Lachnospiraceae;g f_Lachnospiraceae;g__Blautia f_Lachnospiraceae;g_Coprococcus $f$ Lachnospiraceae;g_Lachnobacterium $f$ _Ruminococcaceae; $g$ f_Ruminococcaceae; g_ $f$ Ruminococcaceae;g_Ruminococcus f_Veillonellaceae;g__Dialister f_Veillonellaceae;g_Megasphaera f_Erysipelotrichaceae;g_

\section{B \#OTU ID}

f Bifidobacteriaceae;g Bifidobacterium f Coriobacteriaceae; $g$

f_Coriobacteriaceae;g_Collinsella

f_Bacteroidaceae;g_Bacteroides

$f$ Prevotellaceae; $g$ __Prevotella

f_Enterococcaceae; g_Enterococcus

$f$ Streptococcaceae; $g$ Streptococcus

o_Clostridiales;f_g_

$f$ Clostridiaceae; $g$

f_Clostridiaceae;g_Clostridium

f_Lachnospiraceae; Other

f Lachnospiraceae;g

f_Lachnospiraceae;g__Blautia

f_Lachnospiraceae;g_Coprococcus

f_Lachnospiraceae; g_Lachnospira

$f$ Ruminococcaceae; 9

$f$ Ruminococcaceae;g__Faecalibacterium

f_Ruminococcaceae;g_Ruminococcus

f_Veillonellaceae;g_Megasphaera

f_[Tissierellaceae];g_Peptoniphilus

$f$ Erysipelotrichaceae; $g$ Catenibacterium

f_Enterobacteriaceae; __ f_Lachnospiraceae;g_Lachnospira

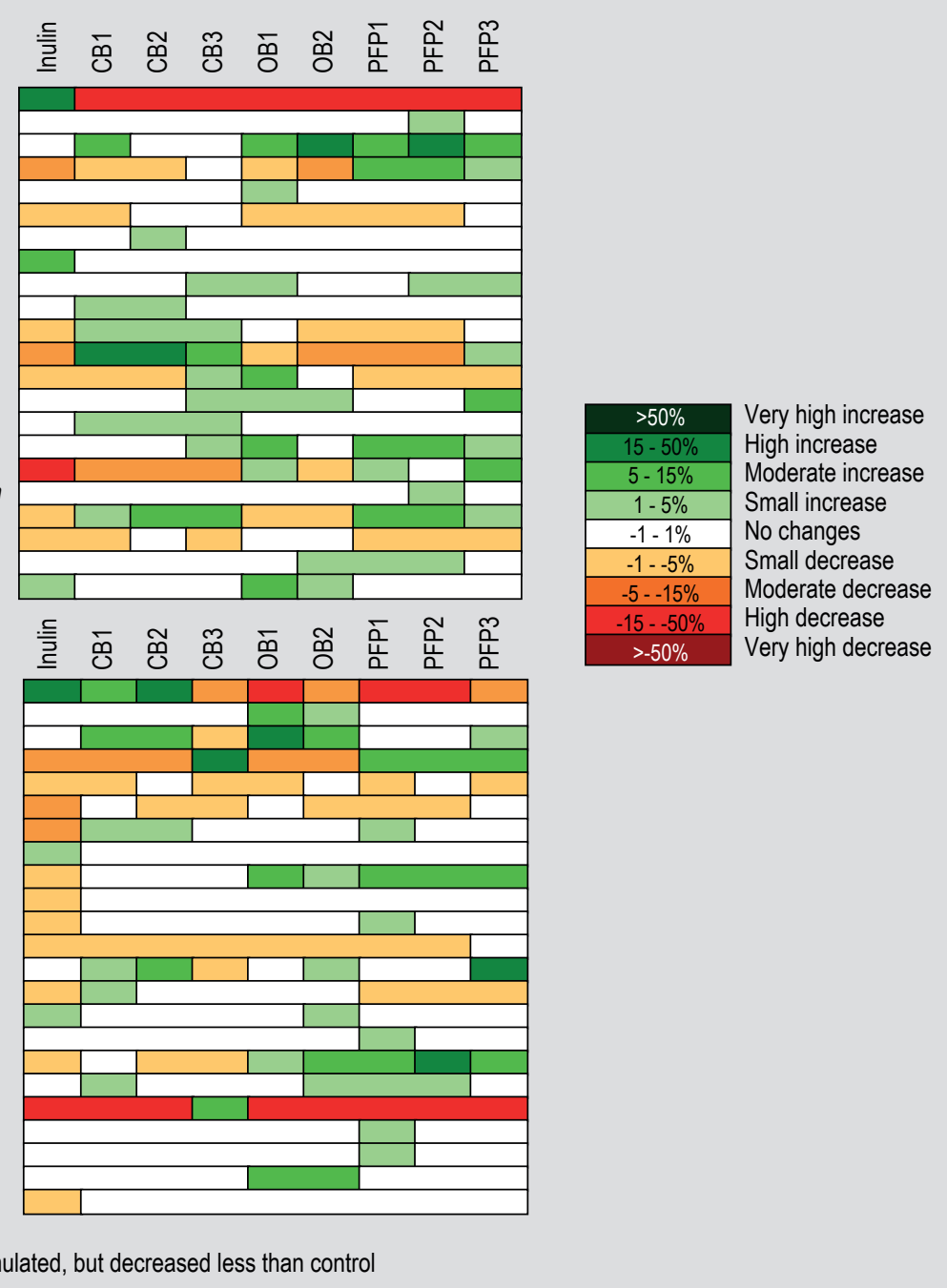

${ }^{*}$ OTUs indicated with a * were not stimulated, but decreased less than control

Figure 5. Heatmap of percentage of changes of bacterial taxa at $t 72 \mathrm{~h}$, compared to control in the lean (A) and obese (B) microbiota. Green colours represent an increase in abundance, whereas red colours represent a decrease. $\mathrm{CB}=\mathrm{cassava}$ bagasse; $\mathrm{OB}=$ orange bagasse; PFP = passion fruit peel. 


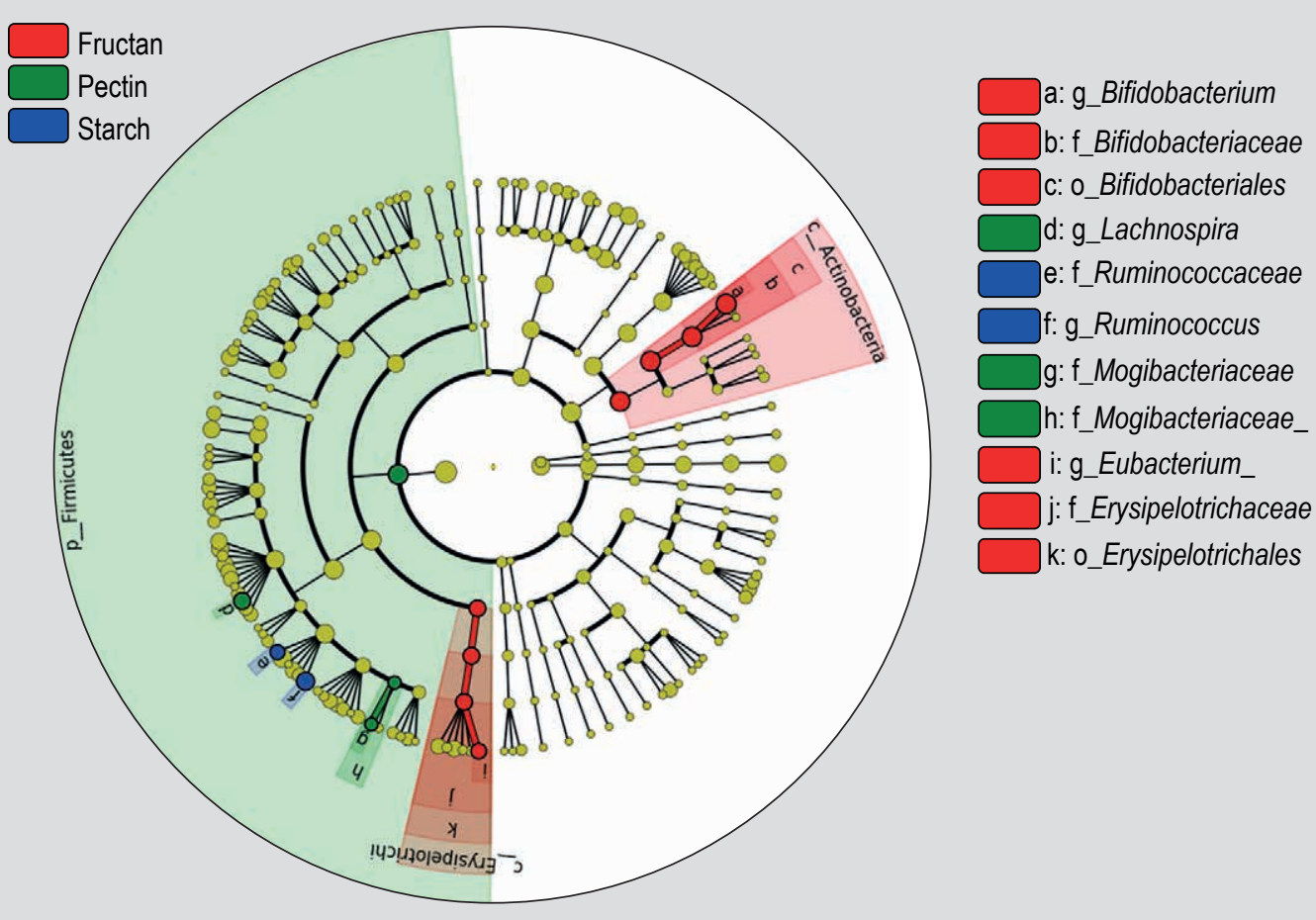

Figure 6. LEfSe with the different primary carbohydrate components showing which bacterial groups were significantly different among samples (lean microbiota).

oligosaccharides, some species are capable of degrading complex carbohydrates like starch (Rajilić-Stojanović and De Vos, 2014), producing mainly acetate and lactate (De Graaf and Venema, 2008). Spearman correlation analyses indicated a positive association between bifidobacteria and the presence of glucose (rho=0.68; q-value $=0.00021)$, the monomer that composes starch. The genus Bifidobacterium is known by its positive correlation with health status of the host and several studies have shown that the genus Bifidobacterium is decreased in several different disease states (Wong et al., 2020).

Interestingly, in our previous study with cassava bagasse, although fermentation of cassava bagasse by the obese microbiota produced an increase in the abundance of Bifidobacterium, a stronger bifidogenic effect was observed by fermentation of the lean microbiota (Bussolo de Souza et al., 2014). Despite some differences between the two studies, still cassava bagasses (CB1 and CB2) were shown here to exert a beneficial effect on the obese microbiota regarding the stimulation of the genus Bifidobacterium.

Cassava bagasses were the substrates that, when used by the obese microbiota, produced similar amounts of acetate compared to our controls, and higher amounts than PFPs $(P<0.05)$ (Figure 3). It was demonstrated in mice that the consumption of vinegar lowered body weight and body fat (Beh et al., 2017). Another study showed a positive effect of acetate related to obesity in overweight and obese men who received infusions of acetate in the distal and proximal colon. Distal infusions of acetate, but not proximal, increased fasting fat oxidation and fasting peptide YY (PYY), when compared to the placebo group (Van der Beek et al., 2016). Another study, nevertheless, showed that increased acetate production by an altered microbiota was found to increase ghrelin secretion, causing hyperphagia and obesity in rats fed with high fat diet (Perry et al., 2016). As mentioned earlier, it seems that the presence of an altered microbiota results in negative effects related to SCFA.

Fermentation of the three tested cassava bagasses enriched the abundance of Ruminococcus in the lean microbiota (Figure 5A). In the obese microbiota, however, fermentation of CB1 and CB2 caused a large decrease in Ruminococcus $(\sim 20 \%)$. CB3, instead, stimulated it by $7 \%$ (Figure $5 \mathrm{~A}$ ), and at $\mathrm{t} 72 \mathrm{~h}$, the microbiota was composed by $30.2 \%$ of the genus Ruminococcus (Figure 7). Some species of Ruminococcus were found to degrade resistant starch (Rajilić-Stojanović and De Vos, 2014). We have demonstrated in an in vitro model of the upper gastrointestinal tract (TIM-1) that CB1, CB2 and CB3 were composed on average of $12 \%$ of resistant starch (Bussolo de Souza et al., 2018), which may explain the reason this genus was enriched upon addition of cassava bagasses. Ruminococcus has been inversely correlated with pro-inflammatory markers, such as interleukin-6 (IL-6) and C-reactive protein (CRP) (Van den Munckhof et al., 2018). 


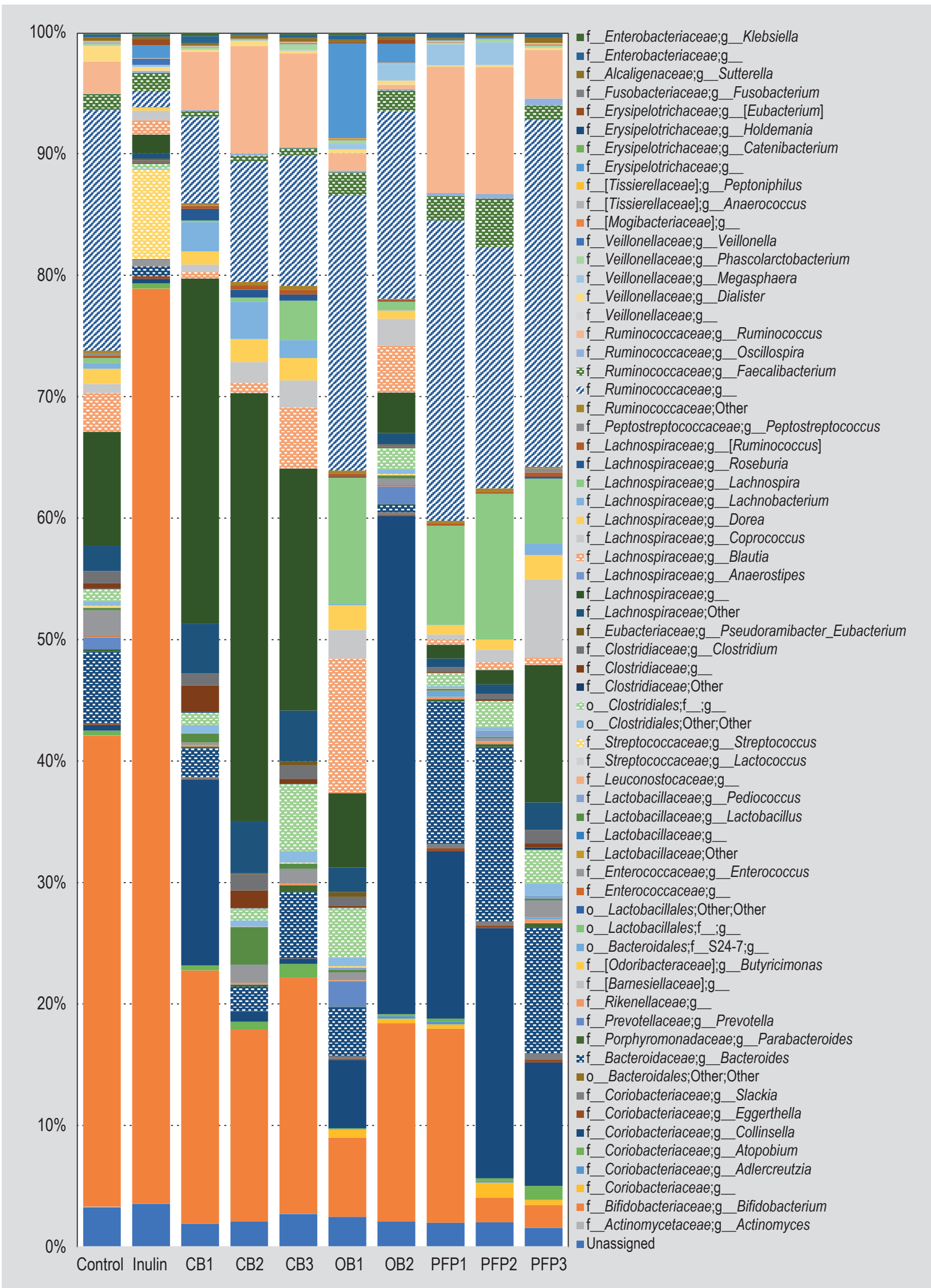

Figure 7A. Microbiota composition after fermentation period with the different substrates (\% of bacterial abundance), in lean microbiota. $C B=$ cassava bagasse; $O B=$ orange bagasse; $P F P=$ passion fruit peel. 


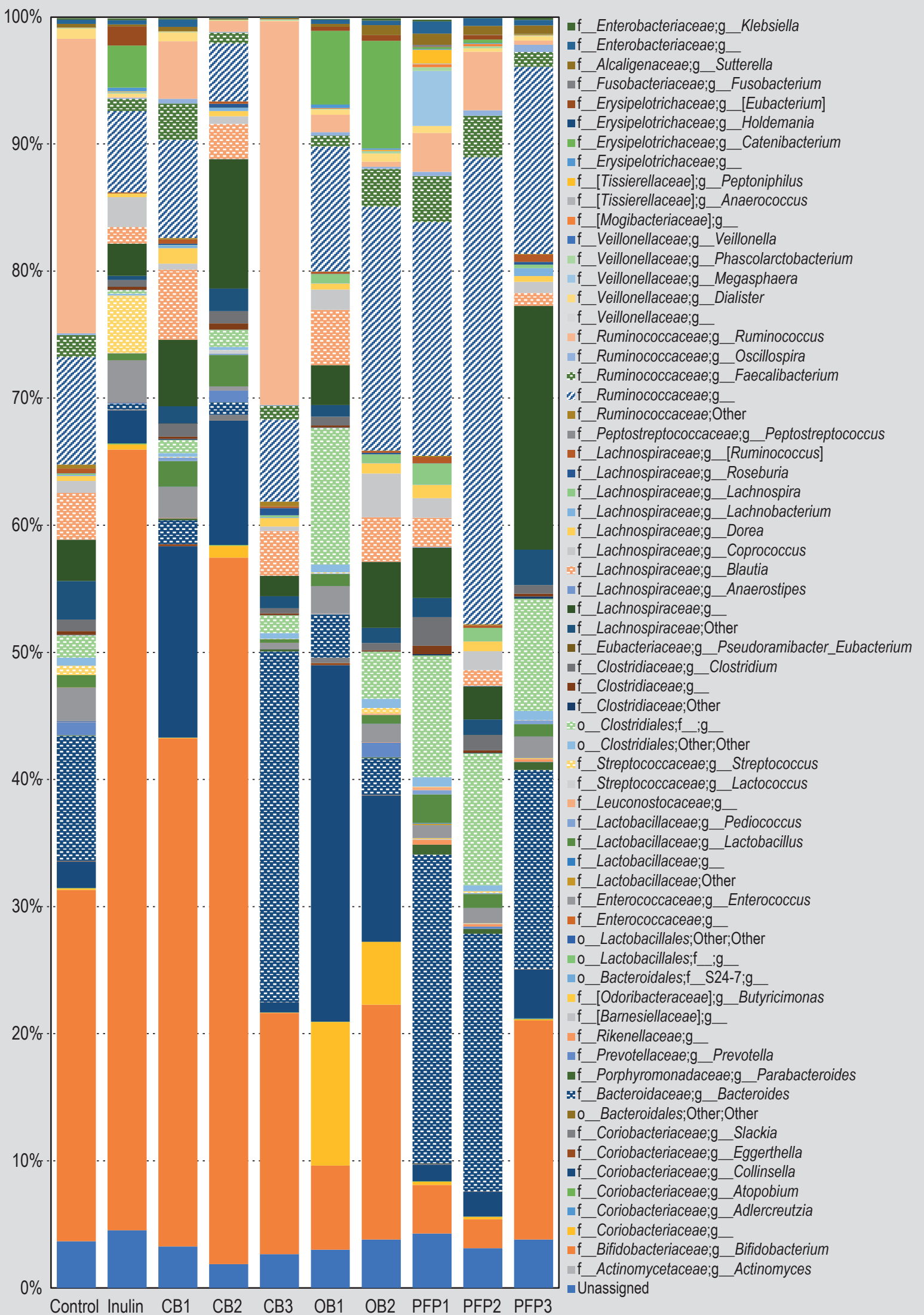

Figure 7B. Microbiota composition after fermentation period with the different substrates ( $\%$ of bacterial abundance), in obese (B) microbiota. $C B$ = cassava bagasse; $O B=$ orange bagasse; $P F P=$ passion fruit peel. 
Obesity is a metabolic disease characterised by a low-grade inflammation, which contributes to an increased risk to develop insulin resistance and type 2 diabetes (Cani et al., 2007). A prebiotic which can enrich the abundance of antiinflammatory species that can contribute to counteracting the negative effects of obesity is highly warranted.

It is very intriguing to observe that cassava bagasses (and passion fruit peels) (Figure 5) were only effective in increasing the abundance of Ruminococcus in lean microbiota. Apart from CB3, all other compounds decreased Ruminococcus abundance of the obese microbiota (Figure 5). It remains unclear what caused such differences.

\section{The butyrate-producing genus Coprococcus was stimulated upon orange bagasses fermentation}

Fermentation of orange bagasses led to the production of SCFA, which levels were comparable to our controls (Figure 3 ), showing the potential of this by-product to be used as substrate by the gut microbiota.

In the lean microbiota, fermentation of $\mathrm{OB} 2$ produced comparable amounts of butyrate $(44.9 \mathrm{mmol})$ to our positive control - inulin $(43.4 \mathrm{mmol})$. In the obese microbiota, fermentation of both orange bagasses - OB1 and OB2 ( $36.9 \mathrm{mmol}$ and $38.5 \mathrm{mmol}$, respectively) resulted in similar amounts of butyrate production as inulin (37.2 $\mathrm{mmol})$. Similar results were found when citrus pectin was used in an in vitro model inoculated with microbiota from obese individuals (Bianchi et al., 2018). Butyrate is by far the most well studied SCFA, and with regard to obesity, it has an important role in keeping the integrity of the intestinal barrier by acting on the mucosal layer and tight junctions (Hatayama et al., 2007; Willemsen et al., 2003). Obesity is characterised by low grade inflammation that might be caused by the translocation of bacterial lipopolysaccharides due to a leaky gut (Cani et al., 2007). It was also demonstrated that acute butyrate administration decreased food intake, while chronic supplementation prevented diet-induced obesity in mice (Li et al., 2018). In our study, butyrate-producing bacteria from the genus Coprococcus were stimulated upon $\mathrm{OB} 1$ and $\mathrm{OB} 1$ addition in the lean microbiota, and by OB2 in the obese microbiota (Figure 5).

A robust increase in abundance of Collinsella upon fermentation of OB2 was found (increase of 40.5\%) with a final abundance of $41 \%$ (Figure 7A), in the lean microbiota. A similar effect of orange bagasses on Collinsella abundance was observed with the obese microbiota. OB1 enriched the abundance of this genus by $26 \%$, and at the end of the fermentation, Collinsella accounted for $28 \%$ of the total abundance (Figure 7B). Although the orange bagasses tested here were rich in small sugars $(22.8 \%$ in OB1 and $33.4 \%$ in OB2) and a positive correlation between Collinsella and small sugars was detected (rho $=0.52$; q-value $=0.03$ ), our previous study with the isolated fibre from orange bagasse (and therefore without small sugars) also found an increase in the genus Collinsella (Bussolo de Souza et al., 2019). Another correlation that may explain such robust increase in this genus due to orange bagasse addition was not found, requiring further research.

A correlation between Collinsella and lipid and glucose metabolism was found in some studies (Gomez-Arango et al., 2016; Lambeth et al., 2015), and Collinsella was found to be increased in subjects that followed a fast food diet (Zhu et al., 2020). The exact mechanism by which Collinsella affects host lipid/glucose metabolism is not completely understood, but it is known that this genus has the ability to deconjugate bile acids (Rajilić-Stojanović and De Vos, 2014). A 'normal' gut microbiota is required for the production of colonic bile acids with higher amounts of secondary bile acids (Tran et al., 2015). Secondary bile acids are ligands of the cell membrane receptor G-protein-coupled bile acid receptor (TGR5), which also triggers the release of the satiety hormones GLP-1 and PYY. These satiety hormones are also recognised for their effects on gut barrier function (Cani et al., 2013).

\section{Lower production of SCFA and intermediate organic acids may be an indication of slow fermentation of passion fruit peels}

PFP1 and PFP2 were the substrates that compared to control and inulin, had the poorest fermentation, yielding smaller amounts of SCFA, although this difference was only significant in the lean microbiota $(P<0.05)$. PFP3, which contained higher amounts of starch among the three passion fruit peels (38.9\%), yielded comparable amounts of total SCFA than control and inulin (Figure 3 and Supplementary Figure S1). Overall, passion fruit peels were the substrates that led to the highest cumulative amounts of BCFA, both in experiments with the lean and obese microbiota (Table 1). PFP1 fermentation by the lean microbiota produced the highest cumulative amount of iso-butyrate $(1.92 \mathrm{mmol})$, whilst in the obese microbiota PFP3 fermentation was responsible for the highest amount of iso-valerate $(3.35 \mathrm{mmol})$.

The passion fruit peels contained higher amounts of proteins, which may explain the production of BCFA, as these are the end-products of bacterial fermentation of proteins and peptides (Flint et al., 2009). Fermentation of passion fruit peels generated negligible amounts of lactate and succinate (Supplementary Table S2), indicating that these by-products were not fermented at a high speed. Lactate and succinate are intermediate metabolites and their accumulation is a sign of fast fermentation (Koenen et al., 2016). 
Table 1. Cumulative production of branched chain fatty acids (iso-butyrate and iso-valerate) by the lean and obese microbiota after $72 \mathrm{~h}$ fermentation with the different substrates. ${ }^{1}$

\begin{tabular}{|c|c|c|c|c|c|c|}
\hline & \multicolumn{2}{|c|}{$i$-butyrate } & \multicolumn{2}{|c|}{$i$-valerate } & \multicolumn{2}{|l|}{ Total } \\
\hline & Lean & Obese & Lean & Obese & Lean & Obese \\
\hline Control & $0.2 \pm 0.2$ & $0.5 \pm 0.8$ & $0.4 \pm 0.1$ & $1.3 \pm 1.2$ & $0.6 \pm 0.3$ & $1.9 \pm 2.0$ \\
\hline Inulin & $0.1 \pm 0.0$ & $0.0 \pm 0.0$ & $0.2 \pm 0.1$ & $0.0 \pm 0.0$ & $0.2 \pm 0.1$ & $0.0 \pm 0.0$ \\
\hline CB1 & $0.7 \pm 0.1$ & $0.7 \pm 0.3$ & $2.1 \pm 0.2$ & $1.5 \pm 0.4$ & $2.7 \pm 0.1$ & $2.2 \pm 0.7$ \\
\hline CB2 & $0.6 \pm 0.2$ & $0.3 \pm 0.0$ & $1.3 \pm 0.2$ & $0.7 \pm 0.0$ & $1.9 \pm 0.5$ & $1.0 \pm 0.1$ \\
\hline CB3 & $1.7 \pm 0.1$ & $0.5 \pm 0.4$ & $1.5 \pm 0.1$ & $0.8 \pm 0.3$ & $3.2 \pm 0.0$ & $1.3 \pm 0.7$ \\
\hline OB1 & $1.2 \pm 0.0$ & $0.2 \pm 0.1$ & $2.2 \pm 0.3$ & $1.2 \pm 0.3$ & $3.4 \pm 0.3$ & $1.4 \pm 0.5$ \\
\hline OB2 & $0.9 \pm 0.1$ & $0.2 \pm 0.2$ & $2.1 \pm 0.0$ & $0.2 \pm 0.0$ & $2.9 \pm 0.1$ & $0.4 \pm 0.2$ \\
\hline PFP1 & $1.9 \pm 0.2$ & $1.8 \pm 0.3$ & $3.3 \pm 0.3$ & $3.0 \pm 0.2$ & $5.2 \pm 0.5$ & $4.8 \pm 0.5$ \\
\hline PFP2 & $1.4 \pm 0.3$ & $0.6 \pm 0.1$ & $2.3 \pm 0.4$ & $1.6 \pm 0.0$ & $3.8 \pm 0.7$ & $2.2 \pm 0.1$ \\
\hline PFP3 & $1.7 \pm 0.1$ & $1.7 \pm 0.7$ & $2.9 \pm 0.4$ & $3.4 \pm 1.0$ & $4.6 \pm 0.5$ & $5.1 \pm 1.8$ \\
\hline
\end{tabular}

Our previous study with isolated fibre from passion fruit peels showed similar results, with lower cumulative production of SCFA and negligible amounts of intermediate metabolites, reinforcing our hypothesis that this by-product was fermented at a slow pace (Bussolo de Souza et al., 2019). This might be a positive feature regarding distal colon health, since most of the fibres are consumed by the gut microbiota in the proximal and transverse part of the colon, and due to the lack of carbohydrates, fermentation of proteins become quantitatively more important distally, where for instance, the majority of colorectal cancers occur (De Graaf and Venema, 2008). Complex composition of passion fruit peels resulted in a microbiota with the highest diversity and evenness (Supplementary Figure S3) among all tested compounds, a positive trait regarding host health.

The genus Bacteroides was stimulated upon fermentation of passion fruit peels, either when the lean or obese microbiota was used. The effects were more prominent for the obese microbiota (Figure 5B), which at the end of the experiment led to higher abundances of Bacteroides than in the lean microbiota (Figure 7). Despite that growth of Bacteroides has been shown to be affected by dilution rate, $\mathrm{pH}$ and other factors (Adamberg and Adamberg, 2018; Adamberg et al., 2020), we show here a positive association between Bacteroides and the presence of xylose (rho=0.79; $\mathrm{q}$-value $\left.=7.09 \times 10^{-06}\right)$ and the presence of fucose $($ rho $=0.63$; q-value $=0.003$ ), both sugars present in PFPs (Supplementary Table S1). Xylose and fucose are sugars present in nonstarch polysaccharides, such as hemicellulose and pectin. However, there was no significant association between Bacteroides and pectin. Other studies demonstrated that Bacteroides was enriched with pectin availability (Aguirre $e t$ al., 2014; Tian et al., 2016). It is worth to highlight, however, that pectins are complex plant hetero-polysaccharides, with major structural differences between various sources (Larsen et al., 2019a). Other characteristics of pectins, like composition of neutral sugars and distribution of homogalacturonan and rhamnogalacturonan fractions might have an effect on the enrichment of diverse bacterial groups. Another in vitro study by us showed that Bacteroides in general was enriched with pectin feeding, but specific species (Bacteroides uniformis, Bacteroides plebeius and Bacteroides ovatus) demonstrated an inconsistent modulation among all different pectins tested (Larsen $e t$ al., 2019b). This is hypothesised to be due to the highly competitive environment, or even specific structural preferences by bacterial groups. This may explain why Bacteroides were only enriched with passion fruit peels and not orange bagasses, although the latter contained higher amounts of pectin (Supplementary Table S1). It appears that other characteristics of pectins are crucial for it to be utilised by Bacteroides. We also found that Bacteroides was positively associated with degree of acetylation (DA) (rho=0.71; q-value=0.0003), and orange bagasses had low DA (Supplementary Table S1).

The genus Faecalibacterium in the lean microbiota only increased in abundance upon PFP2 fermentation, with a final abundance of $4 \%$. In the obese microbiota PFP1 and PFP2 fermentation caused a small increase in Faecalibacterium $(\sim 1.5 \%)$ and the final abundances were $3.7 \%$ and $3.3 \%$, respectively (Figure $7 \mathrm{~B}$ ). Its only known species, F. prausnitizii, is an anti-inflammatory commensal bacterium. Many studies showed inverse correlations between inflammatory bowel disease (IBD) and F. prausnitzii (Machiels et al., 2014; Miquel et al., 2013). 


\section{Conclusions}

Our results showed that fermentation of different types of food-by-products, with different primary carbohydrates, were metabolised differently by the lean and obese microbiota. Regarding microbiota composition, a clear separated cluster at the beginning of the experiments between lean and obese microbiota was found, and at the end of the fermentation period, the clustering within lean and obese samples disappeared, and instead presence of primary carbohydrate in the tested food-by-products drove a new clustering. This new clustering showed the potential of food-by-product in driving the obese microbiota into a healthier profile, similar to lean, with the stimulation of commensal and beneficial bacteria.

The results found in this study show that food-by-products have the potential to be used as a tool to manipulate not only the microbiota from obese individuals, but also from e.g. healthy individuals. The use of food-by-products and the fibres they contain may represent a way to give a better destination to these by-products, such as to incorporate them back into food products as functional ingredients, at the same time increasing fibre intake, which is in general lower than the recommendation. The results of the present mechanistic study show promise that these by-products could be an important step forward to tackle obesity and at the same time decrease the waste of valuable food material and consequently environmental pollution. Moreover, they are inexpensive and a non-invasive way to be used as a dietary intervention to improve health. Further experiments, either in vitro or in vivo are suggested to confirm our findings and contribute to elucidate the interactions between gut microbiota, obesity and utilisation of complex polysaccharides. For instance, combinations of the food-by-products could be tested to study their synergistic or antagonistic effect.

\section{Supplementary material}

Supplementary material can be found online at https://doi. org/10.3920/BM2020.0151.

Figure S1. Kinetics and total cumulative production of short chain fatty acids by the lean and obese microbiota during $72 \mathrm{~h}$ fermentation in TIM-2 with different test compounds.

Figure S2. Total energy production by the lean and obese microbiota after fermentation with the different test compounds.

Figure S3. Shannon index at $\mathrm{t} 72 \mathrm{~h}$ (lean and obese combined) with test compounds.
Table S1. Chemical composition of cassava bagasses, orange bagasses and passion fruit peels.

Table S2. Cumulative production of organic acids (succinate, lactate, formate and caproate) by the lean and obese microbiota after $72 \mathrm{~h}$ fermentation with the different substrates.

\section{Conflict of interest}

The authors declare that there are no conflicts of interest.

\section{Acknowledgements}

Carlota Bussolo de Souza received a PhD grant from CNPq (National Council for Scientific and Technological Development - Brazil) under the program 'Science without Borders' (grant number 246027/2012-6). The study was partly funded by the Centre for Healthy Eating \& Food Innovation (HEFI) of Maastricht University - campus Venlo. This research has been made possible with the support of the Dutch Province of Limburg. We would like to acknowledge Dr. Freddy Troost and Dr. Bouke Salden from Maastricht University for contacting and collecting faecal donation from obese volunteers at Maastricht University Medical Center. Furthermore, we would like to thank the companies that kindly sent samples for this research Alimentos Grazimara, Companhia Lorenz, Chá\&Cia, Phytomare, and Orafti. We thank Dr. Thiago Cardoso de Souza from Rio Grande do Norte Federal University - Brazil - for his assistance with R. We kindly acknowledge Geraldo Bussolo and Zélia Maria Bussolo for collecting samples of orange bagasse and passion fruit peel (OB2 and PFP3) from Brazil. The participation of volunteers that donated their faeces and made this study possible is greatly appreciated.

\section{References}

Adamberg, K. and Adamberg, S., 2018. Selection of fast and slow growing bacteria from fecal microbiota using continuous culture with changing dilution rate. Microbial Ecology in Health and Disease 29: 1549922.

Adamberg, K., Raba, G. and Adamberg, S., 2020. Use of Changestat for growth rate studies of gut microbiota. Frontiers in Bioengineering and Biotechnology 8: 24.

Aguirre, M., Eck, A., Koenen, M.E., Savelkoul, P.H.M., Budding, A.E. and Venema, K., 2015. Evaluation of an optimal preparation of human standardized fecal inocula for in vitro fermentation studies. Journal of Microbiological Methods 117: 78-84.

Aguirre, M., Eck, A., Koenen, M.E., Savelkoul, P.H.M., Budding, A.E. and Venema, K., 2016. Diet drives quick changes in the metabolic activity and composition of human gut microbiota in a validated in vitro gut model. Research in Microbiology 167: 114-125. 
Aguirre, M., Jonkers, D.M.A.E., Troost, F.J., Roeselers, G. and Venema, K., 2014. In vitro characterization of the impact of different substrates on metabolite production, energy extraction and composition of gut microbiota from lean and obese subjects. PLoS ONE 9: e113864.

Anderson, M., 2017. Permutational multivariate analysis of variance (PERMANOVA). Wiley StatsRef. Statistics Reference Online. https://doi.org/10.1002/9781118445112.stat07841

Beh, B.K., Mohamad, N.E., Yeap, S.K., Ky, H., Boo, S.Y., Chua, J.Y.H., Tan, S.W., Ho, W.Y., Sharifuddin, S.A., Long, K. and Alitheen, N.B., 2017. Anti-obesity and anti-inflammatory effects of synthetic acetic acid vinegar and Nipa vinegar on high-fat-diet-induced obese mice. Scientific Reports 7: 6664.

Bianchi, F., Larsen, N., de Mello Tieghi, T., Adorno, M.A.T., Kot, W., Saad, S.M.I., Jespersen, L. and Sivieri, K., 2018. Modulation of gut microbiota from obese individuals by in vitro fermentation of citrus pectin in combination with Bifidobacterium longum BB-46. Applied Microbiology and Biotechnology 102: 8827-8840. https:// doi.org/10.1007/s00253-018-9234-8

Bussolo de Souza, C., Jonathan, M., Isay Saad, S.M., Schols, H.A. and Venema, K., 2018. Characterization and in vitro digestibility of by-products from Brazilian food industry: Cassava bagasse, orange bagasse and passion fruit peel. Bioactive Carbohydrates and Dietary Fibre 16: 90-99.

Bussolo de Souza, C., Jonathan, M., Isay Saad, S.M., Schols, H.A. and Venema, K., 2019. Degradation of fibres from fruit by-products allows selective modulation of the gut bacteria in an in vitro model of the proximal colon. Journal of Functional Foods 57: 275-285.

Bussolo de Souza, C., Roeselers, G., Troost, F., Jonkers, D., Koenen, M.E. and Venema, K., 2014. Prebiotic effects of cassava bagasse in TNO's in vitro model of the colon in lean versus obese microbiota. Journal of Functional Foods 11: 210-220.

Cani, P.D., Amar, J., Iglesias, M.A., Poggi, M., Knauf, C., Bastelica, D., Neyrinck, A.M., Fava, F., Tuohy, K.M., Chabo, C., Waget, A., Delmee, E., Cousin, B., Sulpice, T., Chamontin, B., Ferrieres, J., Tanti, J.-F., Gibson, G.R., Casteilla, L., Delzenne, N.M., Alessi, M.C. and Burcelin, R., 2007. Metabolic endotoxemia initiates obesity and insulin resistance. Diabetes 56: 1761-1772.

Cani, P.D., Everard, A. and Duparc, T., 2013. Gut microbiota, enteroendocrine functions and metabolism. Current Opinion in Pharmacology 13: 935-940.

Caporaso, J.G., Kuczynski, J., Stombaugh, J., Bittinger, K., Bushman, F.D., Costello, E.K., Fierer, N., Pena, A.G., Goodrich, J.K., Gordon, J.I., Huttley, G.A., Kelley, S.T., Knights, D., Koenig, J.E., Ley, R.E., Lozupone, C.A., McDonald, D., Muegge, B.D., Pirrung, M., Reeder, J., Sevinsky, J.R., Turnbaugh, P.J., Walters, W.A., Widmann, J., Yatsunenko, T., Zaneveld, J. and Knight, R., 2010. QIIME allows analysis of high-throughput community sequencing data. Nature Methods 7: 335-336.

De Graaf, A.A. and Venema, K., 2008. Gaining insight into microbial physiology in the large intestine: a special role for stable isotopes. Advances in Microbiological Physiology 53: 73-168.
Flint, H.J., Duncan, S.H. and Louis, P., 2009. Gut microbial ecology. In: McClements, D.J. and Decker, E.A. (eds.) Designing functional foods - measuring and controlling food structure, breakdown and nutrient absorption. Woodhead Publishing, Cambridge, UK, pp. 38-67.

Gibson, G.R., Hutkins, R., Sanders, M.E., Prescott, S.L., Reimer, R.A., Salminen, S.J., Scott, K., Stanton, C., Swanson, K.S., Cani, P.D., Verbeke, K. and Reid, G., 2017. Expert consensus document: The International Scientific Association for Probiotics and Prebiotics (ISAPP) consensus statement on the definition and scope of prebiotics. Nature Reviews Gastroenterology and Hepatology 14: 491-502.

Gomez-Arango, L.F., Barrett, H.L., McIntyre, H.D., Callaway, L.K., Morrison, M. and Dekker Nitert, M., 2016. Connections between the gut microbiome and metabolic hormones in early pregnancy in overweight and obese women. Diabetes 65: 2214-2223.

Hall, K.D., Ayuketah, A., Brychta, R., Cai, H., Cassimatis, T., Chen, K.Y., Chung, S.T., Costa, E., Courville, A., Darcey, V., Fletcher, L.A., Forde, C.G., Gharib, A.M., Guo, J., Howard, R., Joseph, P. V, McGehee, S., Ouwerkerk, R., Raisinger, K., Rozga, I., Stagliano, M., Walter, M., Walter, P.J., Yang, S. and Zhou, M., 2019. Ultraprocessed diets cause excess calorie intake and weight gain: an inpatient randomized controlled trial of ad libitum food intake. Cell Metabolism 30: 67-77.e3.

Hatayama, H., Iwashita, J., Kuwajima, A. and Abe, T., 2007. The short chain fatty acid, butyrate, stimulates MUC2 mucin production in the human colon cancer cell line, LS174T. Biochemical and Biophysical Research Communications 356: 599-603.

Koenen, M.E., Cruz Rubio, J.M., Mueller, M. and Venema, K., 2016. The effect of agave fructan products on the activity and composition of the microbiota determined in a dynamic in vitro model of the human proximal large intestine. Journal of Functional Foods 22: 201-210.

Kovatcheva-Datchary, P., Zoetendal, E.G. Venema, K., De Vos, W.M. and Smidt, H., 2009. Tools for the tract: understanding the functionality of the gastrointestinal tract. Therapeutic Advances in Gastroenterology 2: 9-22.

Lambeth, S.M., Carson, T., Lowe, J., Ramaraj, T., Leff, J.W., Luo, L., Bell, C.J. and Shah, V.O., 2015. Composition, diversity and abundance of gut microbiome in prediabetes and type 2 diabetes. Journal of Diabetes and Obesity 2: 1-7.

Larsen, N., Bussolo de Souza, C., Krych, L., Barbosa Cahú, T., Wiese, M., Kot, W., Hansen, K.M., Blennow, A., Venema, K. and Jespersen, L., 2019a. Potential of pectins to beneficially modulate the gut microbiota depends on their structural properties. Frontiers in Microbiology 10: 223.

Larsen, N., Bussolo de Souza, C., Krych, L., Kot, W., Leser, T.D., Sørensen, O.B., Blennow, A., Venema, K. and Jespersen, L., 2019b. Effect of potato fiber on survival of Lactobacillus species at simulated gastric conditions and composition of the gut microbiota in vitro. Food Research International 125: 108644.

Li, Z., Yi, C.-X., Katiraei, S., Kooijman, S., Zhou, E., Chung, C.K., Gao, Y., Van den Heuvel, J.K., Meijer, O.C., Berbée, J.F.P., Heijink, M., Giera, M., Willems van Dijk, K., Groen, A.K., Rensen, P.C.N. and Wang, Y., 2018. Butyrate reduces appetite and activates brown adipose tissue via the gut-brain neural circuit. Gut 67: 1269-1279. 
Louzada, M.L. da C., Baraldi, L.G., Steele, E.M., Martins, A.P.B., Canella, D.S., Moubarac, J.-C., Levy, R.B., Cannon, G., Afshin, A., Imamura, F., Mozaffarian, D. and Monteiro, C.A., 2015. Consumption of ultra-processed foods and obesity in Brazilian adolescents and adults. Preventive Medicine 81: 9-15.

Machiels, K., Joossens, M., Sabino, J., De Preter, V., Arijs, I., Eeckhaut, V., Ballet, V., Claes, K., Van Immerseel, F., Verbeke, K., Ferrante, M., Verhaegen, J., Rutgeerts, P. and Vermeire, S., 2014. A decrease of the butyrate-producing species Roseburia hominis and Faecalibacterium prausnitzii defines dysbiosis in patients with ulcerative colitis. Gut 63: 1275-1283.

Martens, E.C., Kelly, A.G., Tauzin, A.S. and Brumer, H., 2014. The devil lies in the details: how variations in polysaccharide fine-structure impact the physiology and evolution of gut microbes. Journal of Molecular Biology 426: 3851-3865.

Martinez, R.C., Bedani, R. and Saad, S.M., 2015. Scientific evidence for health effects attributed to the consumption of probiotics and prebiotics: an update for current perspectives and future challenges. British Journal of Nutrition 114: 1993-2015.

Minekus, M., Smeets-Peeters, M., Bernalier, A., Marol-Bonnin, S., Havenaar, R., Marteau, P., Alric, M., Fonty, G. and Huis in't Veld, J.H., 1999. A computer-controlled system to simulate conditions of the large intestine with peristaltic mixing, water absorption and absorption of fermentation products. Applied Microbiology and Biotechnology 53: 108-114.

Miquel, S., Martín, R., Rossi, O., Bermúdez-Humarán, L.G., Chatel, J.M., Sokol, H., Thomas, M., Wells, J.M. and Langella, P., 2013. Faecalibacterium prausnitzii and human intestinal health. Current Opinion in Microbiology 16: 255-261.

Nohr, M.K., Pedersen, M.H., Gille, A., Egerod, K.L., Engelstoft, M.S., Husted, A.S., Sichlau, R.M., Grunddal, K. V, Poulsen, S.S., Han, S., Jones, R.M., Offermanns, S. and Schwartz, T.W., 2013. GPR41/ FFAR3 and GPR43/FFAR2 as cosensors for short-chain fatty acids in enteroendocrine cells vs FFAR3 in enteric neurons and FFAR2 in enteric leukocytes. Endocrinology 154: 3552-3564.

Pandey, A., Soccol, C.R., Nigam, P., Soccol, V.T., Vandenberghe, L.P.S. and Mohan, R., 2000. Biotechnological potential of agro-industrial residues. II: cassava bagasse. Bioresource Technology 74: 81-87.

Perry, R.J., Peng, L., Barry, N.A., Cline, G.W., Zhang, D., Cardone, R.L., Petersen, K.F., Kibbey, R.G., Goodman, A.L. and Shulman, G.I., 2016. Acetate mediates a microbiome-brain- $\beta$ cell axis promoting metabolic syndrome. Nature 534: 213-217.

Rajilić-Stojanović, M. and De Vos, W.M., 2014. The first 1000 cultured species of the human gastrointestinal microbiota. FEMS Microbiology Reviews 38: 996-1047.

Rehman, A., Heinsen, F.-A., Koenen, M.E., Venema, K., Knecht, H., Hellmig, S., Schreiber, S. and Ott, S.J., 2012. Effects of probiotics and antibiotics on the intestinal homeostasis in a computer controlled model of the large intestine. BMC Microbiology 12: 47.

Samuel, B.S., Shaito, A., Motoike, T., Rey, F.E., Backhed, F., Manchester, J.K., Hammer, R.E., Williams, S.C., Crowley, J., Yanagisawa, M. and Gordon, J.I., 2008. Effects of the gut microbiota on host adiposity are modulated by the short-chain fatty-acid binding $G$ protein-coupled receptor, Gpr41. Proceedings of the National Academy of Sciences of the USA 105: 16767-16772.
Sáyago-Ayerdi, S.G., Zamora-Gasga, V.M. and Venema, K., 2020. Changes in gut microbiota in predigested Hibiscus sabdariffa $\mathrm{L}$ calyces and Agave (Agave tequilana Weber) fructans assessed in a dynamic in vitro model (TIM-2) of the human colon. Food Research International 132: 109036.

Scott, K.P., Gratz, S.W., Sheridan, P.O., Flint, H.J. and Duncan, S.H., 2013. The influence of diet on the gut microbiota. Pharmacological Research 69: 52-60.

Segata, N., Izard, J., Waldron, L., Gevers, D., Miropolsky, L., Garrett, W.S. and Huttenhower, C., 2011. Metagenomic biomarker discovery and explanation. Genome Biology 12: R60.

Shin, J.-H., Jung, S., Kim, S.-A., Kang, M.-S., Kim, M.-S., Joung, H., Hwang, G.-S. and Shin, D.-M., 2019. Differential effects of typical Korean versus American-style diets on gut microbial composition and metabolic profile in healthy overweight Koreans: a randomized crossover trial. Nutrients 11: 2450.

Simonnet, A., Chetboun, M., Poissy, J., Raverdy, V., Noulette, J., Duhamel, A., Labreuche, J., Mathieu, D., Pattou, F., Jourdain, M. and the LICORN and the Lille COVID-19 and Obesity study group, 2020. High prevalence of obesity in severe acute respiratory syndrome coronavirus-2 (SARS-COV-2) requiring invasive mechanical ventilation. Obesity 28: 1195-1199. https://doi.org/10.1002/ oby. 22831 .

Tian, L., Scholte, J., Borewicz, K., van den Bogert, B., Smidt, H., Scheurink, A.J.W., Gruppen, H. and Schols, H.A., 2016. Effects of pectin supplementation on the fermentation patterns of different structural carbohydrates in rats. Molecular Nutrition and Food Research 60: 2256-2266.

Tolhurst, G., Heffron, H., Lam, Y.S., Parker, H.E., Habib, A.M., Diakogiannaki, E., Cameron, J., Grosse, J., Reimann, F. and Gribble, F.M., 2012. Short-chain fatty acids stimulate glucagon-like peptide-1 secretion via the G-protein-coupled receptor FFAR2. Diabetes 61: 364-371.

Tran, C.D., Grice, D.M., Wade, B., Kerr, C.A., Bauer, D.C., Li, D. and Hannan, G.N., 2015. Gut permeability, its interaction with gut microflora and effects on metabolic health are mediated by the lymphatics system, liver and bile acid. Future Microbiology 10: 1339-1353.

Turnbaugh, P.J., Ley, R.E., Mahowald, M., Magrini, V., Mardis, E.R. and Gordon, J.I., 2006. An obesity-associated gut microbiome with increased capacity for energy harvest. Nature 444: 1027-31.

Van den Munckhof, I.C.L., Kurilshikov, A., Ter Horst, R., Riksen, N.P., Joosten, L.A.B., Zhernakova, A., Fu, J., Keating, S.T., Netea, M.G., De Graaf, J. and Rutten, J.H.W., 2018. Role of gut microbiota in chronic low-grade inflammation as potential driver for atherosclerotic cardiovascular disease: a systematic review of human studies. Obesity Reviews 19: 1719-1734. https://doi.org/10.1111/obr.12750 Van der Beek, C.M., Canfora, E.E., Lenaerts, K., Troost, F.J., Damink, S.W.M.O., Holst, J.J., Masclee, A.A.M., Dejong, C.H.C. and Blaak, E.E., 2016. Distal, not proximal, colonic acetate infusions promote fat oxidation and improve metabolic markers in overweight/obese men. Clinical Science 130: 2073-2082.

Van der Kamp, J.W., Jones, J., McCleary, B. and Topping, D., 2010. Dietary fibre: new frontiers for food and health. Wageningen Academic Publishers, Wageningen, the Netherlands. 
Willemsen, L.E.M., Koetsier, M.A., Van Deventer, S.J.H. and Van Tol, E.A.F., 2003. Short chain fatty acids stimulate epithelial mucin 2 expression through differential effects on prostaglandin $E_{1}$ and $E_{2}$ production by intestinal myofibroblasts. Gut 52: 1442-1447.

Wong, C.B., Odamaki, T. and Xiao, J., 2020. Insights into the reason of human-residential bifidobacteria (HRB) being the natural inhabitants of the human gut and their potential health-promoting benefits. FEMS Microbiology Reviews 44: 369-385.
World Health Organization (WHO), 2020. Obesity and overweight. Available at: https://tinyurl.com/yxna6hdk

Zhu, C., Sawrey-Kubicek, L., Beals, E., Rhodes, C.H., Houts, H.E., Sacchi, R. and Zivkovic, A.M., 2020. Human gut microbiome composition and tryptophan metabolites were changed differently by fast food and Mediterranean diet in 4 days: a pilot study. Nutrition Research 77: 62-72. 
